\title{
Replication of Two-Dimensional Code Marks Using a Squared Optical Fiber Array
}

\author{
Jun-ya Iwasaki and Toshiyuki Horiuchi \\ Graduate School of Advanced Science and Technology, Tokyo Denki University \\ 5, Senju-Asahi-cho, Adachi-ku Tokyo 120-8551, Japan \\ ik-iwa@d4.dion.ne.jp
}

\begin{abstract}
To develop a simple and low-cost exposure system for the use of code mark printing, projection lithography using an optical fiber array with quasi-square fiber ends was investigated. Plastic optical fibers with a diameter of $1 \mathrm{~mm}$ were reshaped into quasi-squares with a size of $0.92 \mathrm{~mm}$ square and round corners at one end. Printed patterns onto wafers through a projection lens with a reduction ratio of $1 / 10$ were also round at corners. To evaluate the corner roundness, square ratio $S$ was newly defined and calculated. $S$ was 0 when the patterns were real circles, and 1 when they were squares, respectively. It was clarified that square ratio became large at the focused positions and under the appropriate exposure dose conditions. $S$ was always smaller than that calculated for the shape of the optical fiber. This is probably caused by the roundness of fiber corners and the lack of light irradiance homogeneity at the fiber ends.
\end{abstract}

Keyword: optical fiber, square end, projection lithography, code mark, square ratio, exposure system, light emitting diode

\section{Introduction}

Improvement of yields is very important to reduce product costs and obtain expected product volumes timely. For this reason, makers of electronic devices and liquid crystal display (LCD) panels print serial numbers and code marks on each product and use them for the production managements [1][2].

Projection exposure systems equipped with super high-pressure mercury lamps have been used as conventional exposure tools to print serial numbers and code marks, and the management data have been assigned by small LCD panels used as reticles [3]. However, mercury lamps are very expensive in spite of their short life spans of approximately one month [4][5]. In addition, because the LCD panels used as reticles have low transmittance or reflectance for the exposure light, only some parts of the light energy are used.

For this reason, it is required that a simple and highly efficient exposure system to print serial numbers and code marks is promptly developed. In such situation, an idea of exposure system using light emitting diode (LED) arrays as exposure sources was contrived. However, because an LED emits light at a small point in various directions, it is difficult to print patterns with square shapes in narrow pitches and intervals. For this reason, a new method was contrived to print square patterns without spaces between the patterns [6].

In the new method, light emitted from each LED is collected by a lens and injected to an 
optical fiber, and the other end of the fiber is reshaped into a square. Thus, square secondary sources are made, and they are combined in an array without making gaps [7][8].

In this paper, patterning characteristics of square patterns printed by using the optical fiber are investigated. Light leaks at the squared fiber corners, if the fibers are deformed in squares with sharp right angles. For this reason, round parts are left at each corner [9]. In addition, because light rays lead in the circular parts are divided in the square region, irradiance homogeneity in the square cross section may degrade comparing with that in the circular one [10]. Worrying about these subjects, shape accuracy dependence of square patterns on exposure time and defocus is investigated [11][12].

\section{Projection Exposure System Using Squared Optical Fibers}

Test exposure system using LED-array sources and squared optical fibers was fabricated, as shown in Fig. 1.

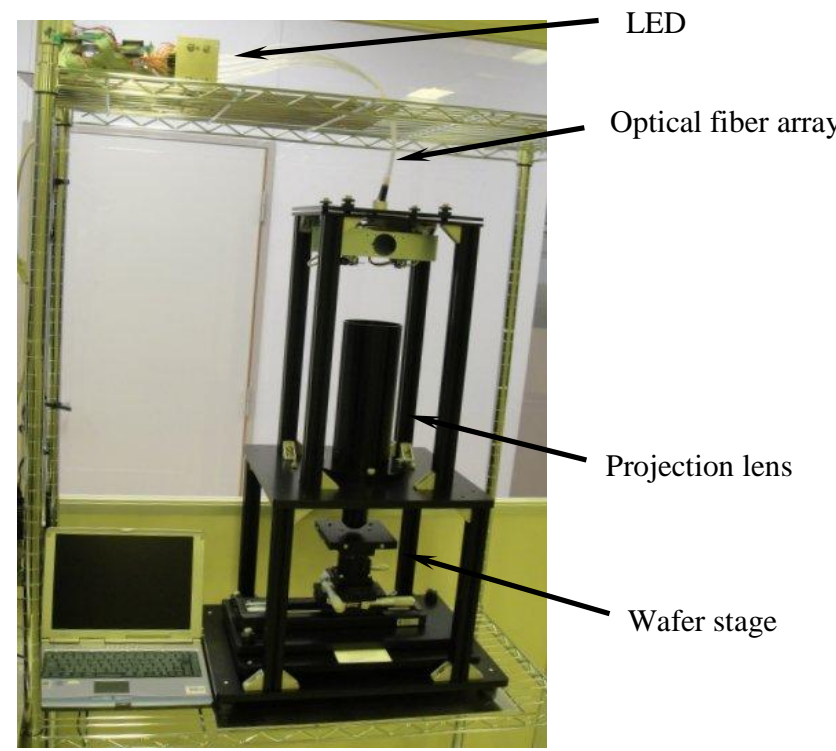

Fig. 1. Exposure system using LED-array sources and squared optical fibers.

As exposure sources, LEDs with a wavelength of $405 \mathrm{~nm}$ (Opto Supply LED, OSSV5111A) were used. The central wavelength was $405 \mathrm{~nm}$. Light rays emitted from each LED was collected by a lens, and introduced into an optical fiber, as shown in Fig. 2.
Plastic optical fibers with a diameter of 1 $\mathrm{mm}$ were used, and the outlet ends were reshaped into $0.92 \mathrm{~mm}$ quasi-squares in $5 \mathrm{~mm}$ long from the ends, as shown in Fig. 3.

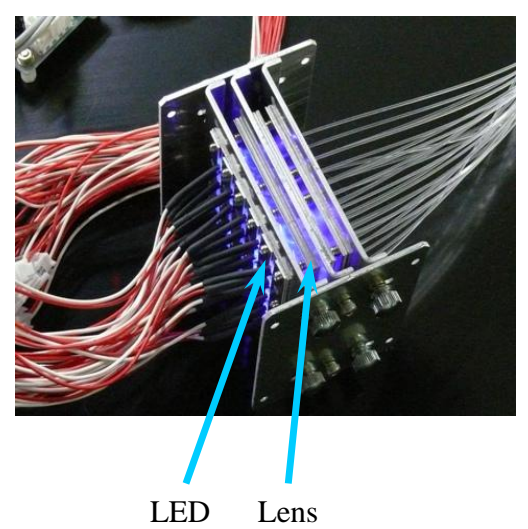

Fig. 2. Light collection optics placed between an LED and an optical fiber.

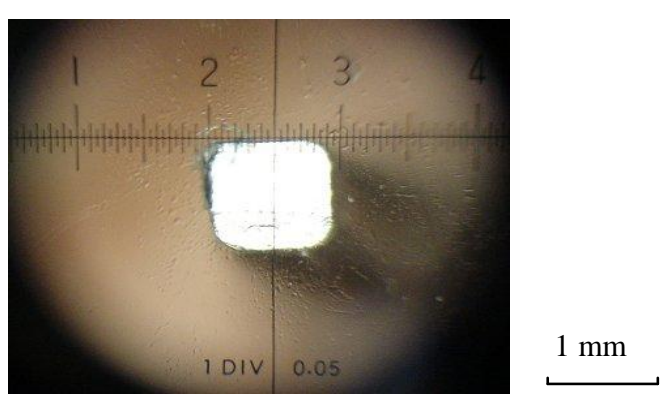

Fig. 3. Cross section of squared optical fiber.

The exits of fibers were projected by a lens with a reduction ratio of $1 / 10$ onto wafers.

Figures 4 shows the ejection end of fibers arrayed in $5 \times 5$ matrix, and Fig. 5 shows the LED illumination controller to assign the LEDs to be lit on and off.

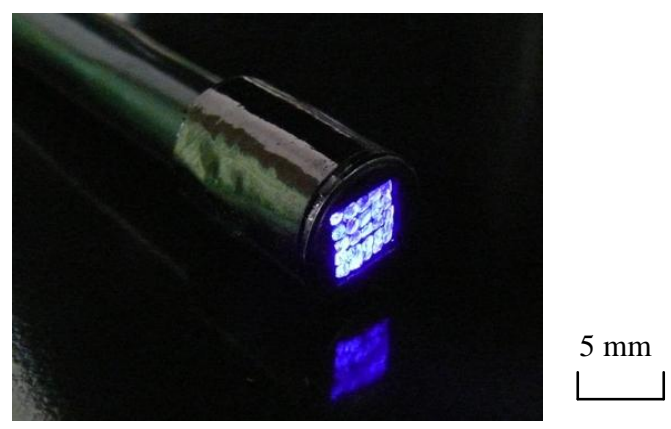

Fig. 4. Light ejection end with squared fibers. 


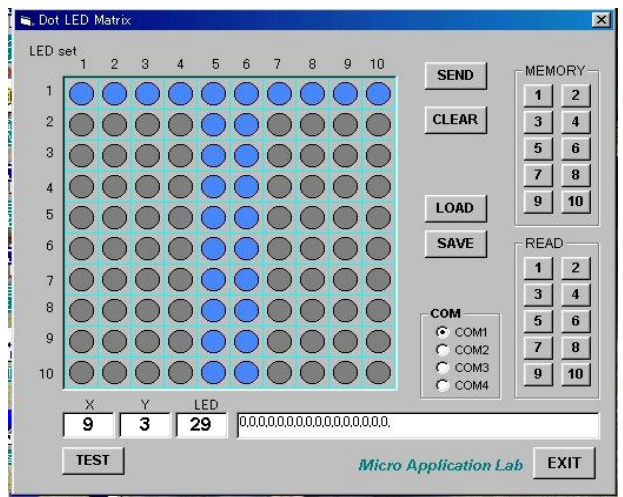

Fig. 5. LED illumination controller.

\section{General Patterning Characteristics}

Using a set of LED and fiber with a cross section shown in Fig. 3, patterning characteristics were investigated. Silicon wafers with a diameter of 4 " were coated with a positive resist THMR-ip3300 (Tokyo Ohka Kogyo) in $0.25 \mu \mathrm{m}$ thick.

Figures 6 shows the relationship between the replicated pattern profile and the focal position, and Figure 7 shows the pattern size dependence on the focal position for various exposure times.

Pattern sizes were measured in both horizontal and vertical directions, and the mean values were plotted.

It was clarified that pattern size was almost constant for $\pm 20 \mu \mathrm{m}$ focal position ranges, if appropriate focal positions were selected.

\begin{tabular}{|l|l|l|l|}
\hline \multicolumn{5}{|c|}{ Focal Position $(\mu \mathrm{m})$} \\
\hline-70 & -30 & -10 & 0 \\
\hline & & & \\
\hline & & & \\
\hline
\end{tabular}

$100 \mu \mathrm{m}$

Fig. 6. Pattern profile change depending on the focal position.

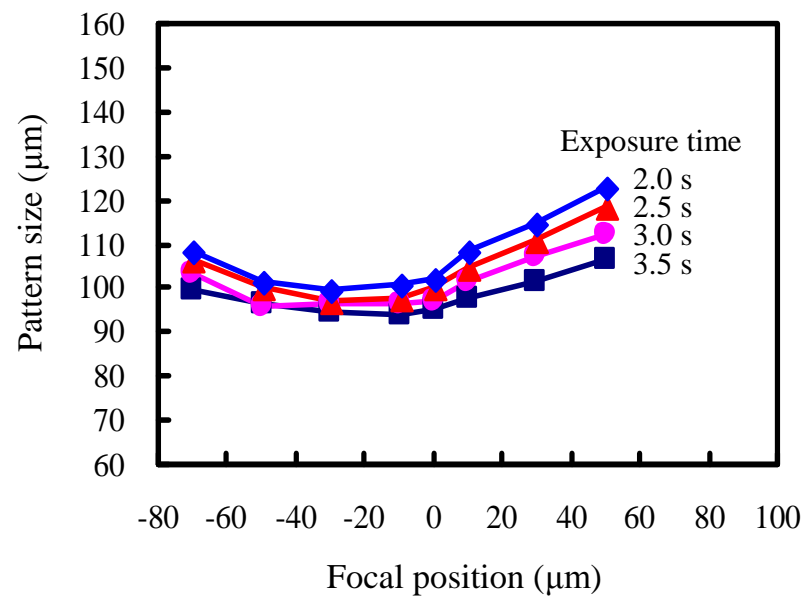

Fig. 7. Relationship between pattern size and focal position.

On the other hand, Fig. 8 shows the pattern size dependence on the exposure time.

The exposure time was sufficiently short to prospect actual applications. Exposure dose tolerance to control the pattern sizes within $\pm 5 \%$ was as long as $\pm 0.5 \mathrm{~s}$ for the exposure time of $2.2 \mathrm{~s}$.

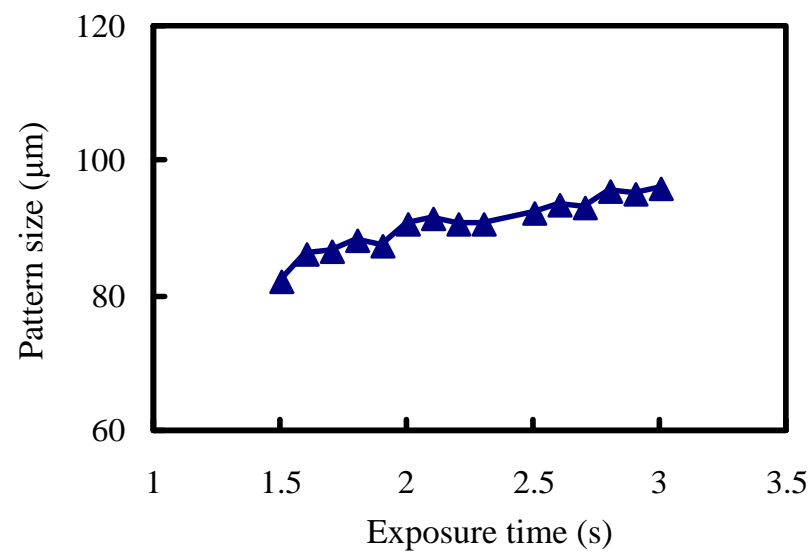

Fig. 8. Relationship between pattern size and exposure time.

\section{Investigation of Square Shapes}

Then, deformations of square pattern shapes were investigated. Figure 9 explains the deformation of optical fiber. It was considered that differences between $a_{1}, a_{2}$ and $c_{1}, c_{2}$ were related to the corner sharpness.

For this reason, following parameter was defined as the "square ratio" $S$. 


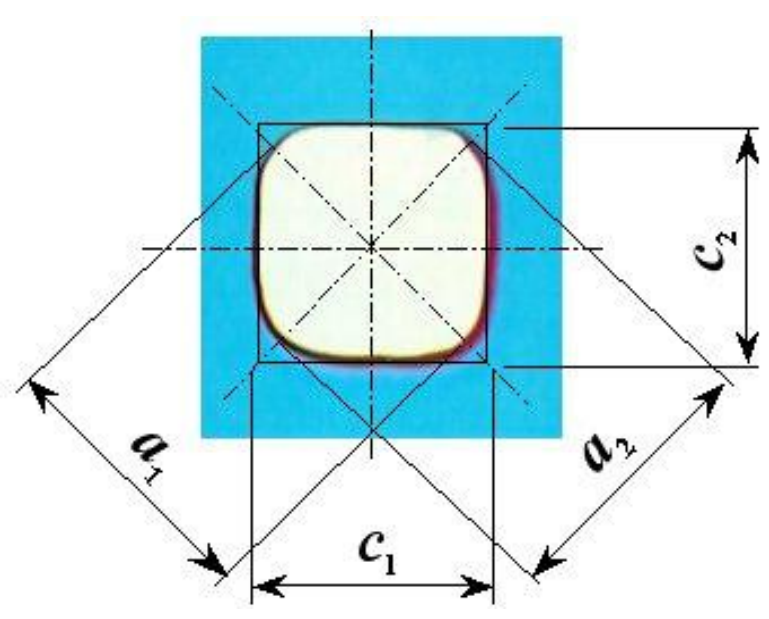

Fig. 9. Deformation of optical fiber.

$$
S=\frac{1}{\sqrt{2}-1} \frac{\frac{a_{1}+a_{2}}{2}-\frac{c_{1}+c_{2}}{2}}{\frac{c_{1}+c_{2}}{2}}=\frac{1}{\sqrt{2}-1} \frac{a-c}{c}
$$

Here,

$$
\begin{aligned}
& a=\frac{a_{1}+a_{2}}{2} \\
& c=\frac{c_{1}+c_{2}}{2}
\end{aligned}
$$

When $a$ equals to $c, S$ becomes 0 , and the pattern shape becomes to a circle. On the other hand, if the pattern shape is a real square, $S$ becomes 1 .

The parameter $S$ was calculated for the patterns obtained by the experiments. However, because the original fiber end had not a real square shape, calculated $S$ was as small as less than 0.4. For this reason, relative square ratio $S$ ' was calculated, next. Here, $S$ ' was normalized by the square ratio $S_{0}$ of the fiber end, as shown below.

$$
S^{\prime}=S / S_{0}
$$

Square ratio of the fiber ends was

$$
S_{0}=0.500
$$

and calculated results are shown in Figs. 10 and 11 .

Relative square ratio of $S$ ' widely changed depending on the defocus, and $S^{\text {' }}$ was most stable and large at the defocus of
$-30 \mu \mathrm{m}$, as shown in Fig. 10. On the other hand, $S$ ' did not change much depending on the exposure time.

Therefore, the main parameter deciding the square ratio is the focal position. However, $S$ did not reach to $S_{0}$. This is probably because the light irradiance distribution at the fiber end is not homogeneous. It is supposed that the irradiance at near the corners is lower than that at the center and at side center. Light rays are expanded in larger areas at the square corners. On the other hand, light rays are collected in smaller areas at side centers.

It should be investigated in detail that the present patterns are applicable to code marks or not. Probably, the shape of the fiber end and the length of the squared part had better be improved some more.

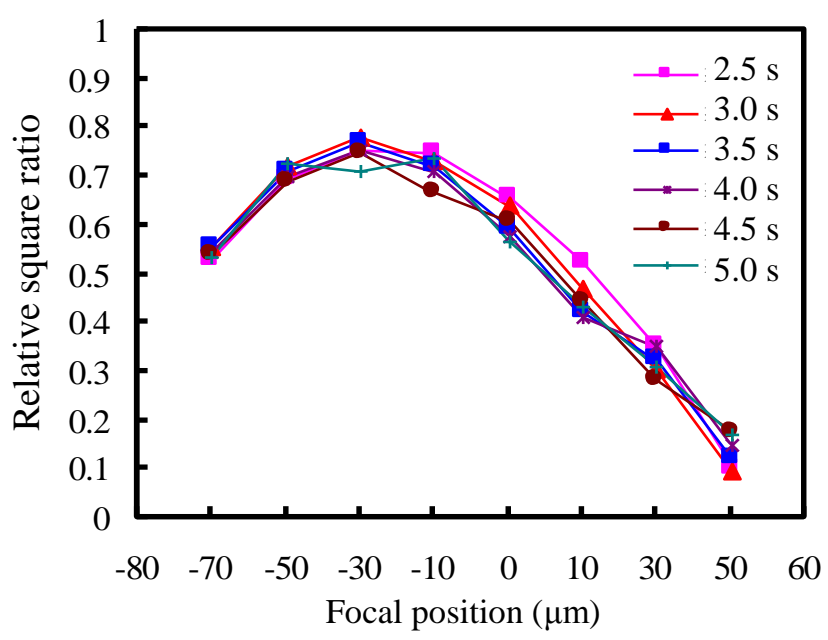

Fig. 10. Relationship between square ratio and focal position.

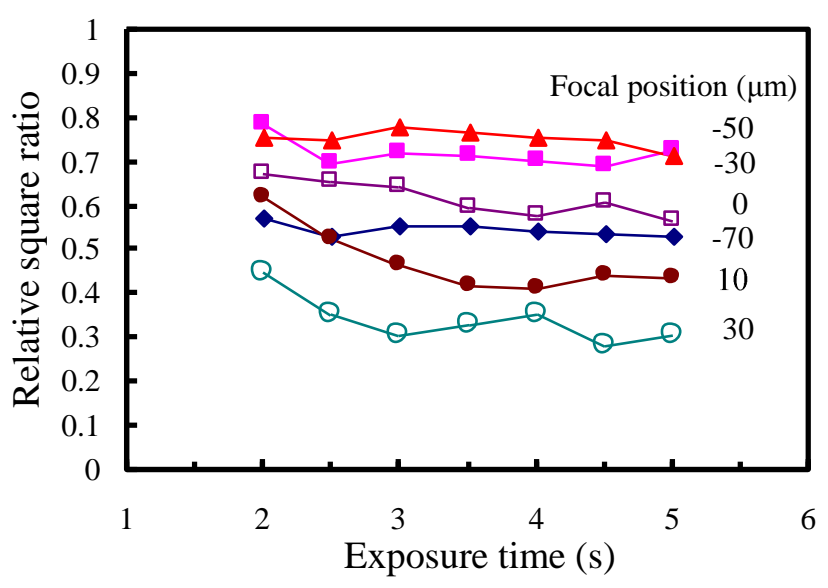

Fig. 11. Relationship between square ratio and exposure time. 


\section{Conclusion}

Projection exposure, using optical fibers with an exit shape of quasi-square, was investigated. Such exposure is effective to print code mark patterns on wafers and substrates of display panels.

Although square patterns are preferable for 2 dimensional code marks, patterns had round corners. For this reason, differences from real square were investigated. Because the original shapes of the source optical fiber ends were round at the corners, the shape differences between the fiber end the patterns were evaluated.

As a result, square ratios of the patterns were always smaller than that of the optical fiber end. This is probably caused by the lack of irradiance homogeneity at the quasi-square fiber exit.

Because the light rays are widely expanded at corners and collected at side centers, the square ratio probably decreases depending on the irradiance distribution. The shape of the fiber end and the length of the squared part should be improved some more.

\section{Acknowledgement}

This work was partially supported by Research Institute for Science and Technology of Tokyo Denki University Grant Number Zc 08-01 in 2008 and Q 09M-05 in 2009.

\section{References}

1. J. Iwasaki and T. Horiuchi: Ext. Abst. $7^{\text {th }}$ International Conference Optics-photonics Design \& Fabrication (ODF 2010, Yokohama) 341-342.

2. J. Iwasaki, K. Ishikawa, Y. Higashida, S. Msatsui, T. Horiuchi: Ext. Abst. Autumn Meeting 2008, The Japan Society for Precision Engineering, 557 [in Japanese].

3. T. Horiuchi and A.Ohtani: OPTICAL REVIEW 14 (2007) 224-230.

4. M. Kase and T. Sawa: J. Illum. Eng. Inst. Jpn. 88 (2004) 853-861 [in Japanese].

5. M. Kase and T. Sawa: J. Illum. Eng. Inst. Jpn. 89 (2005) 473-481 [in Japanese].

6. J. Iwasaki and T. Horiuchi, Ext. Abst. (The 53rd Spring Meeting, 2006), The Japan Society of Applied Physics and Related Societies, p811 (in Japanese).

7. S. H. Tu, J. W. Pan, C. M. Wang, Y. C. Lee, and J. Y. Chang: OPTICAL REVIEW 16 (2009) 318-322.

8. Y. Oki, S. Miyawaki, M. Maeda and M. Tanaka: OPTICAL REVIEW 12 (2005) 301-306.

9. F. M. Schellenberg: OPTICAL REVIEW $\mathbf{1 2}$ (2005) 83-89.

10. A. Serebriakov, E. Maksimov, F. Bociort and J. Braat: OPTICAL REVIEW 12 (2005) 140-145.

11. A. Motogaito, N. Machida, T. Morikawa, K. Manabe, H. Miyake, and K. Hiramatsu: OPTICAL REVIEW 16 (2009) 455-457.

12. S. H. Tu, S. Y. Wu, Y. C. Lee, J. W. Pan, C. H. Kuo, C. M. Wang, and J. Y. Chang: OPTICAL REVIEW 17 (2010) 379-384. 